\title{
Patient Centered Care and Professional Nursing Practices
}

\author{
Mehad Araki* \\ Tawam hospital, SEHA, AlAin, United Arab Emirates
}

\begin{abstract}
Patient-centered care (PCC) has become a key focus in the delivery of health care and providing care centered on patients' needs and expectations is a key attribute of quality care. The goal of patient centered care is to empower patients to become active participants in their care. PCC is a measure of the quality of health care and understanding the characteristics of PCC facilitates its implementation and measurement. This requires health care providers to address patient needs effectively.

Patient-centered care also requires that the health care provider become a patient advocate and strive to provide care that not only is effective but also safe. Researches has shown that promoting patient centered care activities will improve patients' adherence, promote patients' responsibilities for health status and lead to improved health outcomes.

This article provides more clarity to the concept of patient centered care, its dimensions, and benefits in relation to both patients and health care organizations. It also considers the contribution of nurses to PCC and in the enhancement of service delivery.

Kevwords: Patient Centered Care. Nursing. Patients.
\end{abstract}

\section{Introduction}

Patient centered care (PCC) is an essential determinant of quality care. Patient centered care is a multidimensional concept, and institutions that adopt PCC approach report better patient and organizational outcomes. Patient-centered care focuses the attention on patient's beliefs, choices, preferences, and needs, in contrast to physician centered care [1]. PCC has been shown to result in better health outcomes, better survival, greater patient satisfaction and wellbeing, however, little is still known about the importance of PCC aspects and dimensions [2].

Despite growing recognition of the importance of patient centered care, and its effectiveness, studies show that patients report significant problems in gaining access to information, understanding treatment options, getting explanations regarding medications, and receiving responsive service from the health care professional [3].

The paper discusses the concept of patient centered care, its dimensions, benefits in relation to both patients and health care organizations and the professional nursing practices related to patient centered care.

\section{Patient Centered Care}

There is no universally agreed upon definition of patient centered care; however, Richardson and his colleagues [4] defined PCC as care that is "respectful of and responsive to individual patient preferences, needs, and values, and ensuring that patient values guide all clinical decisions." Patient Centered Care represents a shift from traditional, paternalistic, provider-driven, disease focused approaches towards healthcare systems that respects patients' preferences, needs, desires and experiences. PCC empowers patients, and enable health care providers to partner with patients to better meet patient goals [5].

Corresponding Author: Mehad Araki, Tawam hospital, SEHA, AlAin, United Arab Emirates Received date: September 22, 2019; Accepted date: October 01, 2019; Published date: October 02, 2019.

DOI: https://doi.org/10.31546/JBRCI.1004 
Contemporary health care has evolved tremendously since the concept of patient center care was introduced in the late 1980s. The concept of PCC has received increased attention in recent years and is now considered to be an essential inspiration of high quality health care systems [6].

Patient centered care occurs when all the actions and processes are directed toward reducing and eliminating patient vulnerabilities. During hospitalization, patients are vulnerable not only because of compromised physiologic states but also because of threats to their personal identity (such as perceiving a lack of control or being unable to decide for themselves). Vulnerabilities are addressed and lessened by therapeutic engagement of the patient in the process of planning and implementation of care. Successful therapeutic engagement is achieved by developing trust and through interactions between patients and health care professionals. When therapeutic engagement is successful, the patient receives effective care, needs are met, and suffering is lessened [7].

\section{Dimensions of Patient Centered Care}

The Picker Institute identified eight dimensions of PCC. Theses dimensions were discovered through researches by the Picker Institute and Harvard Medical School. The researches involved gathering information from diverse focus groups including patients, physicians, heath care professionals and family members regarding their health care related experiences, and reviewing relevant literature to determine what matters most to patients.

The dimensions are (1) respect for patient preferences, values and expressed needs; (2) coordination and integration of care and services; (3) information, education and communication; (4) emotional support; (5) physical comfort; (6) involvement of family and friends; (7) continuity and transition; and (8) access to care and services [8].

\section{Respect for Patients' Values, Preferences, and Expressed Needs}

The first dimension talks about treating patients with respect, maintains their dignity and demonstrates sensitivity to their cultural beliefs and values. It also emphasize on keeping patients informed about their condition and involving them indecision making process. This dimension focuses on the patients' quality of life, which may be affected by their illness and treatment [8].

\section{Coordination and Integration of Care}

Coordinating and integrating patients' care help to reduce feelings of anxiety, fear and vulnerability. Patients identified three areas in which care coordination can reduce feelings of vulnerability; (1) coordination of clinical care,
(2) coordination of ancillary and support services and (3) coordination of front line patient care were identified by patients [8].

\section{Information and Education}

Providing complete information to patients regarding their health status, treatment options, progress, and prognosis help ensure their autonomy and their ability to make decisions about their health [8].

\section{Physical Comfort}

This dimension focuses on promoting patients' physical comfort with regard to pain management, support with the activities of daily living, and maintaining a focus on the hospital environment (e.g., privacy, cleanliness, comforts, accessibility for visits) [8].

\section{Emotional Support and Alleviation of Fear and Anxiety}

The fifth dimension promote alleviation of fear and anxiety the person may be experiencing with respect to their health statute, the impact of their illness on themselves and others and the financial impacts of their health conditions [8].

\section{Involvement of Family and Friends}

Acknowledging and respecting the role of the person's family and friends in their health care is achieved by: accommodating the individuals who provide the person with support during care, respecting the role of the person's advocate in decision making and supporting family members and friends as caregivers, and recognizing their needs as well [8].

\section{Continuity and Transition}

This dimension of PCC ensure eliminating anxiety about the patients' ability to self-manage after discharge by providing information regarding medication, physical restrictions, nutrition. It also focuses on coordinating ongoing treatment and services and sharing this information with patients and their families [8].

\section{Access to Care}

Patients should be assured about and made aware of their access to ambulatory care, access to specialists and specialty services when needed, health care settings and services, and availability of transportation. Patients should be informed about scheduling process and availability of appointments [8]. As understanding of these dimensions by the healthcare professionals is essential for delivering patient centered, a study was 
conducted to investigate the perspectives of healthcare professionals of the eight dimensions of patient centered care. [2]. Thirty four healthcare professionals working at a large teaching hospital in New York City were interviewed. Participants were asked to rank 35 statements representing the eight dimensions of PCC. Result showed that not all dimensions of PCC were equally important for PCC as perceived by the health care professional. Three main dimensions of PCC were identified; treating patients with dignity and respect, an interdisciplinary approach, and equal access and good outcomes. This study showed that healthcare professionals did not perceived all eight dimensions of PCC as equally important for the improvement of such type of care. The study concluded that viewpoints on essential elements for patient centered care appeared to differ among health care professionals [2].

\section{Benefits of Patient Centered Care}

Patient centered care views patient as an active participant who should be involved in any decision making processes. The shift to PCC enhances concordance between health care professionals and patients' adherence to treatment plans, thus increasing patients' satisfaction with healthcare services. PCC practices have resulted in significant benefits for patients, as they can better manage their health when they are involved, engaged and supported [9].

Bertakis and Azari [10] showed that a PCC approach had significantly reduced patients need to access health care ( $\mathrm{p}<$ 0.0209), hospitalizations ( $\mathrm{p}<0.0033$ ) and required fewer investigations $(p<0.0027)$. Similarly, the adoption of a PCC approach in the management of patients with chronic hypertension led to an increase in medication adherence (RR $3.19,95 \%$ CI 1.44, 16.23) [11]. Other benefits related to patient centered care, include improving patients' selfperceptions, reducing stress and increasing empowerment, and have also been reported in diabetes mellitus management [12].

Organization that adopt patient centered care approaches as in cardiac and respiratory rehabilitation helped patients to make lifestyle modifications, improve their overall health and wellbeing and enhance their experiences $[13,14,15]$. PCC has also shown to decrease the lengths of hospital stays and readmission rates, enhanced primary health care services and improved patients' functional capacities. The outcomes of PCC approach suggests benefits to both patients and healthcare organizations. [16].

A clinical randomized study was conducted to examine whether patient centered care (PCC) impacts patient satisfaction, perception of nursing care, and quality of care. Differences were seen in 2 of 3 subscales within the Baker and
Taylor Measurement Scale. The PCC group rated satisfaction $(\mathrm{P}=.04)$ and quality of services $(\mathrm{P}=.03)$ higher than controls. PCC may impact patients' perception of the level of satisfaction and quality of care received [17].

The Australian Quality Framework for Health Care (ACSQHC) identifies patient centered care as an essential component of any safe and high quality health care system. The integration of PCC approaches in clinical areas has resulted in a number of changes to clinical services as seen in clinical bedside handovers and an increased presence of family members during medical rounds). Traditionally, clinical handovers occurred away from patients and patients were not viewed as being integral to their own healthcare [18].

The introduction of bedside handovers promotes communication in relation to patient's clinical status and care planning. It also provides patients with an opportunity to take a part and ask questions about their health. The Australian Quality Framework for Health Care (ACSQHC) and the World Health Organization (WHO) identified this initiative as being key to improving patients' safety. Including patients in the process of transferring information regarding their health status, has enhanced the accuracy of information and communication. [18,19].

An Australian study showed that participation and involvement in handovers improved patients' safety and enhanced the satisfaction of both patients and nursing staff. It was noted that over $44 \%$ of nurses perceived improvements in patient safety as a result of the handovers [20]. Thus, including patients in clinical handovers enhances treatment decision making processes, increases feelings of empowerment, improves functional outcomes and reduces the use of ancillary health services [21].

Moreover, discharge planning improved patients' outcomes, as they were able to identify the key considerations being made in relation to the planning and coordination of support services [20]. Patients who participate in their own care are more likely to observe, identify and communicate potential issues; thus, eliminating risks and promoting the safety and quality of care provided [22].

\section{Patient Centered Care and Professional Nursing Practices}

The implementation of PCC among healthcare workers is an essential part in daily work. 
The literature showed that as the nursing staff is 24 hours a day at the bedside of the patient, they play the most significant role in PCC implementation [23].

Currently health care organization focuses in shifting from the previous approach which was medically dominated and disease orientated to a patient centered approach. The PCC approach acknowledges patients' values and enhance their engagement and involvement in the decisions making process. Patient centered care is inherent to many of nursing care theories such as Leininger's (1988) theory of culture care [24], Boykin and Schoenhofer's (1993) theory of nursing as caring [25] and Roach's (1987) conceptualization of caring relationships [26].

In 2014 The Israeli Ministry of Health conducted national survey of patient care experience in general hospitals. In this survey 11,098 patients from 24 hospitals were interviewed. The results of the survey showed that nursing staff has a crucial influence on the patients care experience, their satisfaction and perception of being in the center of care [27].

Given this background, one of the main nursing efforts is to identify patients' needs and problems. This requires that the patients' interpretations of nursing care be examined. Certainly, patient centered care requires nurses to ask patients about their perception of care and the importance of prioritizing various nursing care activities. Nurses can't ensure that their behavior is consistent with caring needs of their patients. Also, they cannot assume that patients perceive caring efforts as they are intended. To avoid such problems and to ensure patient centered care, it is important that nurses validate with the patients that their caring needs are being met. Moreover, nurses have to pay attentions to all aspects of nursing care that patients consider to be important [28].

Developing a health care workforce that is committed to patient centered care involves creating a supportive work environment for employees and engaging them in the design, implementation and evaluation of PCC processes [29,30]. Health care professional should be vital in the evaluation of patient centered care as their perspectives and experiences can provide important information for quality improvement, and their engagement can stimulate improvements in ensuring high quality care [31].

One of the most important elements in PCC implementation is the training of nursing staff on PCC. The nursing training programs should provide nurses with tools to identify the unique needs of each patient, as well as provide tools for shared decision making. One of the important tools for this is advanced communication skills [32].

Journal of Biomedical Research and Clinical Investigation
Teaching nurses about PCC should not only be in classroom teaching, but also through simulations that examine the student's ability to implement the principles of PCC in practice [33]. In order for care processes to be directed toward reducing vulnerabilities, nurses should know the patients and adapt the plan of care to meet their, perspectives, beliefs, and values. The meaning that illness may have to that particular patient should also be considered. The skills, knowledge, and competencies of the nurses influence patients' ability to achieve their goal and alleviate vulnerabilities [7]. Furthermore, individualized comprehensive nursing care should respect and focuses on emphasizing views and wishes of patients. Nurses need to ensure that they are providing care according to what the patients want and not what the nurses want. [28].

\section{Conclusion}

Patient centered care is a model of care that respects the patient's values, needs, choices and preferences in the planning process and implementation of his or her care. Patient centered care model has been shown to contribute to improved outcomes for patients and organization, decreased costs and increased satisfaction with care [34]. Healthcare organizations looking forward to improve patient centered care have to consider the importance of its dimensions in their specific context of care provision, which will improve levels of patient centeredness in a more efficient, effective and focused manner [2].

Health care providers should acknowledge primary ethical principles to ensure that patients are provided with the information they need to make decisions and are supported in any decision making processes. Extensive research has been conducted on the benefits attributable to patient centered care and indicates that it can result in outcomes that benefit both patients and health care organizations. Thus, when PCC is provided with support, education and information, patients are capable of appropriately applying this information to better manage their health and evaluate their own health status. (35).

\section{Conflict of interest}

The author declares no conflict of interests.

\section{References}

1. Jayadevappa R. Patient-Centered Outcomes Research and Patient-Centered Care for Older Adults: A Perspective. Gerontol Geriatr Med. 2017; 3: 2333721417700759. 
2. Berghout $\mathrm{M}$, van Exel J, Leensvaart L, Cramm JM. Healthcare professionals' views on patient-centered care in hospitals. BMC Health Serv Res. 2015 ;15:385.

3. Shaller D. Patient-Centered Care: What Does It Take? The Commonwealth Fund.2007.

4. Institute of Medicine (US) Committee on Quality of Health Care in America. Crossing the quality chasm: a new health system for the 21st century. National Academies Press (US). 2001.

5. Bokhour BG, Fix GM, Mueller NM, et al. How can healthcare organizations implement patient-centered care? Examining a large-scale cultural transformation. BMC Health Serv Res. 2018; 18:168.

6. Greene SM, Tuzzio L, Cherkin D. A framework for making patient-centered care front and center. Perm J. 2012; 16:49-53.

7. William BJ. The way to patient-centered care. Nursing Management: Picker Institute. 1987; 41:10-12.

8. Picker Institute. Principles of patient-centered care. (1987).

9. Delaney LJ. Patient-centred care as an approach to improving health care in Australia. Collegian. 2018. 25:119-123.

10. Bertakis KD. Azari R. Patient-centred care is associated with decreased health care utilization. J Am Board Fam Med. 2011; 24:229-39.

11. Roumie CL, Greevy R, Wallston KA, et al. Patient centred primary care is associated with patient hypertension medication adherence. J Behav Med. 2011; 34:244-53

12. Hermanns N, Kulzer B, Ehrmann D, et al. The effect of a diabetes education programme (PRIMAS) for people with type 1 diabetes: Results of a randomized trial. Diabetes Res Clin Pract. 2013; 102:149-157.

13. Cegala DJ, Street RL Jr, Clinch CR. The impact of patient participation on physicians' information provision during a primary care medical interview. Health Commun. 2007; 21:177-85.

14. Coulter A, Parsons S. Askham J. Where are the patients in decision-making about their own care? World Health Organization Regional Office for Europe. Denmark. 2008.

15. Robinson JH, Callister LC, Berry JA, Dearing KA. Patient-centered care and adherence: definitions and applications to improve outcomes. J Am Acad Nurse Pract. 2008; 20:600-607.

16. Hermanns N, Kulzer B, Ehrmann D, Bergis-Jurgan N, Haak T. The effect of a diabetes education programme (PRIMAS) for people with type 1 diabetes: Results of a randomized trial. Diabetes Res Clin Pract. 2013; 102:14957.

17. Wolf DM, Lehman L, Quinlin R, Zullo T, Hoffman L. Effect of Patient-Centered Care on Patient Satisfaction and Quality of Care. J Nurs Care Qual. 2008; 23:316-321.
18. Australian Commission on Safety and Quality in Health Care (ACSQHC). Patient charter of rights, Sydney, Australia: ACSQHC. 2007.

19. World Health Organisation. People-centred health care: A policy framework. Geneva, Switzerland: World Health Organization. 2007.

20. Chaboyer W, McMurray A, Johnson J, et al. Bedside handover: Quality improvement strategy to 'transform care at the bedside'. J Nurs Care Qual. 2009; 24:136-42.

21. Sidani S, Epstein D, Miranda J. Eliciting patient treatment preferences: A strategy to integrate evidence-based and patient-centered care. Worldviews Evid Based Nurs. 2006; 3:116-23.

22. Broom M, Brady B, Kecskes, Z, Kildea S. World Café methodology engages stakeholders in designing a Neonatal Intensive Care Unit. J Neonatal Nurs. 2013; 19:253-258.

23. Merav BN., Ohad H. Patient-Centered Care in Healthcare and its Implementation in Nursing. International Journal of Caring Sciences. 2017; 10:569-600.

24. Leininger MM. Leininger's theory of nursing: Cultural care diversity and universality. Nurs Sci Q. 1988; 1:152-160.

25. Boykin A, Schoenhofer S. Nursing as caring: A model for transforming practice. NLN Publ. 1993 ;(15-2549):i-xxxii, 1-108.

26. Roach MS. The human act of caring. Ottawa, Canada: Canadian Hospital Association. 1987;163.

27. Aka ZA, Greenbaum AA. Binder BI. (2015) Survey of patient in departments in general hospitals.2015.

28. Azimzadeh, R, Valizadeh L, Zamanzadeh V, Rahmani A. What are important for patient centered care? A quantitative study based on perception of patients' with cancer. J Caring Sci. 2013; 2: 321-327.

29. Leggat SG, Bartram T, Casimir G, Stanton P. Nurse perceptions of the quality of patient care: Confirming the importance of empowerment and job satisfaction. Health Care Manage Rev. 2010; 35:355-64.

30. Rathert C, May DR.. Health care work environments, employee satisfaction, and patient safety: Care provider perspectives. Health Care Manage Rev. 2007; 32:2-11.

31. Balbale SN, Turcios S, LaVela, SL. Health care employee perceptions of patient-centered care. Qual Health Res. 2015; 25:417-425. 
32. Smith-Stoner M. Teaching PCC during the Silver Hour. Online J Issues Nurs. 2011; 16:6.

33. Onge JLS, Parnell RB. Patient centered care and patient safety: a model for nurse educators. Teaching and Learning in Nursing. 2015; 10:39-43.

34. Gluyas H. Patient-centred care: improving healthcare outcomes. Nurs Stand. 2015. 30:50-57.

35. Delaney LJ. Patient-centred care as an approach to improving health care in Australia. Collegian. 2018; 25:119-123. 